\title{
On the Theory of Fermionic Preheating
}

\author{
Patrick B. Greene \\ Department of Physics, University of Toronto, 60 St. George Street, Toronto, ON M5S 1A7, Canada \\ Lev Kofman \\ CITA, University of Toronto, 60 St. George Street, Toronto, ON M5S 1A7, Canada
}

(October 15, 2018)

\begin{abstract}
In inflationary cosmology, the particles constituting the Universe are created after inflation due to their interaction with moving inflaton field(s) in the process of preheating. In the fermionic sector, the leading channel is out-of equilibrium particle production in the non-perturbative regime of parametric excitation, which respects Pauli blocking but differs significantly from the perturbative expectation. We develop theory of fermionic preheating coupling to the inflaton, without and with expansion of the universe, for light and massive fermions, to calculate analytically the occupation number of created fermions, focusing on their spectra and time evolution. In the case of large resonant parameter $q$ we extend for fermions the method of successive parabolic scattering, earlier developed for bosonic preheating. In an expanding universe parametric excitation of fermions is stochastic. Created fermions very quickly, within tens of inflaton oscillations, fill up a sphere of radius $\simeq q^{1 / 4}$ in monetum space. We extend our formalism to the production of superheavy fermions and to 'instant' fermion creation.
\end{abstract}

\begin{tabular}{lll} 
PACS: $98.80 . \mathrm{Cq}$ CITA-2000-05 hep-ph/0003018 \\
\hline
\end{tabular}

\section{INTRODUCTION}

During cosmic inflation, it is assumed that entropy and temperature associated with particles of matter are diluted to practically zero values together with the number density of particles. After inflation, the inflaton field $\phi$ oscillates around the minimum of its effective potential $V(\phi)$. The energy of inflaton oscillations is converted to the energy of newly created particles of matter. Eventually particles of different species are settled in a state of thermal equilibrium which marks the beginning of the conventional epoch of the hot Friedmann radiation domination. The actual process of particle creation from the classical background inflaton oscillations occurs very rapidly in the regime of parametric resonance [1] before the thermal equilibrium will be settled. Particles are created non-perturbatively in the outof-equilibrium state. The theory of this process, preheating, is elaborated in details for the creation of bosons [2. For bosons (denoted $\chi$ ), the leading effect is the stimulated process of particle creation in the regime of parametric resonance, where the number density of created particles copiously increases with time as $n_{\chi} \sim \exp \int \mu d t$. Soon, the backreaction of created $\chi$ particles becomes important, so that the self-consistent dynamics of interacting bose fields $\phi(t, \vec{x})$ and $\chi(t, \vec{x})$, which can be treated classically, can be revealed with lattice simulations [3].

At the beginning of the preheating investigation, a study of fermion production did not look very interesting relative to the bosonic case. Indeed, the number density of fermions $\psi$ is bounded by Pauli blocking. Therefore, it was not expected that fermions will influence the dynamics of the inflaton and other scalar fields (despite a numerical study [6] which down-played an observation that fermion production from an oscillating scalar is different from the perturbative prediction).

However, it was understood with surprise [4,5] that creation of fermions from the coherently oscillating inflaton field occurs very differently than what the conventional perturbation theory of inflaton decay $\phi \rightarrow \bar{\psi} \psi$ (say, due to a Yukawa coupling $h \bar{\psi} \phi \psi$ ) would predict, and this may have many interesting cosmological applications. It turns out that the occupation number of fermions very quickly, within about ten inflaton oscillations, is saturated at the time-average value of about $n_{\psi} \sim 1 / 2$. Moreover, in momentum space, fermions are excited within a non-degenerate "Fermi sphere" of a large radius $k \sim q^{1 / 4} m$, where $m$ is the frequency of inflaton oscillations, $q$ is the usual dimensionless parameter of parametric excitation, $q=\frac{h^{2} \phi_{0}^{2}}{m^{2}}$. Ironically, it may be parametric excitations of fermions that are responsible for the most important observable signatures or observational constraints of preheating. Indeed, in some inflationary models there is significant production of gravitinos during the preheating stage [11. Gravitinos are cosmologically dangerous relics, for a mass of $\sim 100 \mathrm{Gev}$ their abundance relative to that of the relic photons cannot exceed the bound $n_{3 / 2} / n_{\gamma} \leq 10^{-15}$. Theory of gravitino production from preheating is rooted in the theory of spin $1 / 2$ fermionic preheating. Another potentially important application of fermionic preheating is a possibility to produce superheavy fermions with a mass as large as $\sim 10^{18} \mathrm{Gev}$ from inflatons of mass $10^{13} \mathrm{Gev}$, as was noticed in [7]:8] and investigated 
in [8]. Superheavy fermions may be interesting for the dark matter problem and for the problem of ultra-high energy cosmic rays. There are other interesting cosmological applications for the creation of fermions, e.g. the scenario of instant preheating [15] and the creation of massive fermions during inflation [14]. Recently, fermionic preheating in hybrid inflation for some range of parameters was thoroughly studied 12 . At present, it is hard to say how important fermionic preheating will be in the self-consistent non-linear dynamics of bose- and fermi-fields during preheating. Technical difficulty is to incorporate fermions into lattice simulations alongside with bosons. However, in [4] it was reported that even within the Hartree approximation backreaction of fermions catalyzes bosonic preheating. After all, for complete decay of inflatons one needs a 'three-legs' interaction, which is provided naturally by the Yukawa coupling with fermions.

In this paper we develop the theory of fermionic preheating in an expanding universe, following our short paper [5], with an emphasis on the analytic results. In particular, we will generalize for the fermionic case some of the methods which we earlier developed for bosonic preheating [2],9], in particular, the method of parabolic scattering, which works for large values of $q$, gives us an analytic formula for the occupation number of fermions $n_{k}(t)$ as function of time and momentum. We extend this method for production of superheavy fermions from a moving scalar field. We begin Sect. 2 of this paper with the Dirac equation and different VEVs for fermions interacting with a time-dependent background scalar field. In Sect. 3 the creation of fermions without expansion of the universe will be considered. We mostly will consider scalar fields oscillating in a potential $V=\frac{m^{2} \phi^{2}}{2}$, although the methods can easily be applied to others. We will give a semi-analytic treatment of the problem based on some earlier results. For the case $q \gg 1$ we develop the method of successive parabolic scatterings. In Sect. 4 we take into account expansion of the universe, when preheating of fermions acquires new qualitative features. We extend the theory to describe the production of superheavy fermions. Our formalism also includes the case when fermions are created not from inflaton oscillations, but from a single instance, when inflaton field crosses a certain level.

\section{FORMALISM: FERMIONS COUPLING WITH BACKGROUND SCALAR IN FRW METRICS.}

We will consider the creation of spin- $\frac{1}{2}$ Dirac fermions $\psi$ by a homogeneous, oscillating scalar field $\phi$ in an expanding, flat FRW universe. Our strategy will be to solve the Heisenberg equation of motion for the quantum $\psi$-field in the presence of the classical backgrounds $\phi$ and $g_{\mu \nu}$. Furthermore, we will assume the energy-momentum of the $\phi$-field alone determines the expansion rate of the Universe. The matter action we use

$$
S_{\mathrm{M}}\left[\phi, \psi, e_{\mu}^{\alpha}\right]=\int d^{4} x e\left[\frac{1}{2} \partial_{\mu} \phi \partial^{\mu} \phi-V(\phi)+i \bar{\psi} \bar{\gamma}^{\mu} \vec{D}_{\mu} \psi-\left(m_{\psi}+h \phi\right) \bar{\psi} \psi\right]
$$

contains a simple Yukawa coupling between the scalar field with effective potential $V(\phi)$ and fermion with baremass $m_{\psi}$. Here $\bar{\gamma}^{\mu}$ is a space-time dependent Dirac gamma matrix, $e_{\mu}^{\alpha}$ is the vierbein with $e$ its determinant, and

$D_{\mu}=\partial_{\mu}+\frac{1}{4} \gamma_{\alpha \beta} \omega_{\mu}^{\alpha \beta}$ is the spin- $\frac{1}{2}$ covariant derrivative with vierbein dependent spin-connection, $\omega_{\mu}^{\alpha \beta}$. The unbarred gammas are standard, Minkowski space-time Dirac matrices and $\gamma_{\alpha \beta} \equiv \gamma_{[\alpha} \gamma_{\beta]}$.

\section{A. Classical Background Fields}

We consider flat FRW metrics $d s^{2}=d t^{2}-a(t)^{2} d \vec{x}^{2}$ where $a(t)$ is the scale factor of the universe. The Hubble parameter $H \equiv \frac{\dot{a}}{a}$ is determined by the equation $H^{2}=\frac{8 \pi}{3 M_{\mathrm{pl}}^{2}}\left(\frac{1}{2}(\dot{\phi})^{2}+V(\phi)\right)$. The background homogeneous scalar field obeys the equation of motion $\ddot{\phi}+3 H \dot{\phi}+\frac{\partial V}{\partial \phi}=0$. We will be most interested in fermion creation while the $\phi$-field oscillates about a minimum of its potential. For illustration of the methods, we will consider fermion creation in the context of chaotic inflation scenarios with potentials of the form $V(\phi)=\frac{1}{2} m_{\phi}^{2} \phi^{2}+\frac{\lambda}{4} \phi^{4}$. After inflation, the $\phi$-field oscillates quasi-periodically with a slowly decreasing amplitude due to the Hubble expansion. The specific value of the parameter $m_{\phi}$ or $\lambda$ (or some combination thereof) will be dictated by cosmology. We mostly will consider quadratic potential with $\lambda=0$. In this paper we ignore backreaction of created particles.

\section{B. Quantum Dirac Field}

Variation of the action (11) with respect to $\bar{\psi}$ leads to the general relativistic generalization of the Dirac equation

$$
\left[i \bar{\gamma}^{\mu} D_{\mu}-\left(m_{\psi}+h \phi(t)\right)\right] \psi(x)=0
$$


It can be shown that for an FRW space-time we have $\bar{\gamma}^{0}=\gamma^{0}$ and $\bar{\gamma}^{i}=\frac{1}{a(t)} \gamma^{i}$, where the $\gamma^{\alpha}$ 's are standard, Minkowski space-time Dirac matrices. Furthermore, spin connection leads to

$$
\frac{1}{4} \bar{\gamma}^{\mu} \gamma_{\alpha \beta} \omega_{\mu}^{\alpha \beta}=\frac{3}{2}\left(\frac{\dot{a}}{a}\right) \gamma^{0}
$$

Thus, in our case, the Dirac equation becomes

$$
\left[i \gamma^{0} \partial_{0}+i \frac{1}{a} \vec{\gamma} \cdot \vec{\nabla}+i \frac{3}{2}\left(\frac{\dot{a}}{a}\right) \gamma^{0}-\left(m_{\psi}+h \phi\right)\right] \psi(x)=0 .
$$

Only standard gamma matrices now appear. Similarly, $\bar{\psi}=\psi^{\dagger} \gamma^{0}$ is found to obey the conjugate of Equation (4).

In the Heisenberg representation of QFT, the Dirac equation (2) or (4) becomes the equation of motion for the $\psi(x)$ field operator. $\psi(x)$ may be decomposed into eigen-spinors

$$
\psi(\mathbf{x}, t)=\sum_{s= \pm} \int \frac{d^{3} k}{(2 \pi)^{3}}\left(\hat{a}_{k, s} \mathbf{u}_{k, s}(t) e^{+i \mathbf{k} \cdot \mathbf{x}}+\hat{b}_{k, s}^{\dagger} \mathbf{v}_{k, s}(t) e^{-i \mathbf{k} \cdot \mathbf{x}}\right),
$$

where $\mathbf{u}_{k, \pm}(t)$ is a positive-frequency eigenspinor of the Dirac equation (4) with helicity $\pm \frac{1}{2}$ and $\mathbf{v}_{k, \pm}(t)=\mathcal{C} \overline{\mathbf{u}}_{k, \pm}^{T}(t)$ is its charge conjugate. Here $\mathcal{C}=i \gamma_{0} \gamma_{2}$ is the standard charge conjugation matrix. To construct these eigen-spinors we use the ansatz

$$
\mathbf{u}_{k, \pm}(t) e^{+i \mathbf{k} \cdot \mathbf{x}}=a\left[-i \gamma^{0} \partial_{0}-i \frac{1}{a} \vec{\gamma} \cdot \vec{\nabla}-i \frac{3}{2}\left(\frac{\dot{a}}{a}\right) \gamma^{0}-\left(m_{\psi}+h \phi\right)\right] \chi_{k}(t) R_{ \pm}(\mathbf{k}) e^{+i \mathbf{k} \cdot \mathbf{x}}
$$

where $R_{ \pm}(\mathbf{k})$ are eigenvectors of the helicity operator $\mathbf{k} \cdot \boldsymbol{\Sigma}$ such that $\mathbf{k} \cdot \boldsymbol{\Sigma} R_{ \pm}(\mathbf{k})= \pm 1$ and $\gamma^{0} R_{ \pm}(\mathbf{k})=+1$. The time dependence of the eigen-spinor is contained intirely in the mode function $\chi_{k}(t)$. Substituting this ansatz into equation (4) leads to the mode equation

$$
\ddot{\chi}_{k}+4 \frac{\dot{a}}{a} \dot{\chi}_{k}+\left[\frac{k^{2}}{a^{2}}+m_{\mathrm{eff}}^{2}-i \frac{\left(a \dot{M}_{\mathrm{eff}}\right)}{a}+\frac{9}{4}\left(\frac{\dot{a}}{a}\right)^{2}+\frac{3}{2} \frac{\ddot{a}}{a}\right] \chi_{k}=0,
$$

where $k^{2}=|\mathbf{k}|^{2}$ and the effective mass of the fermions is

$$
M_{\mathrm{eff}} \equiv\left(m_{\psi}+h \phi\right) .
$$

The damping term in this equation may be removed by defining a new mode function $X_{k}(t)=a^{2} \chi_{k}(t)$. The mode equation becomes

$$
\ddot{X}_{k}+\left[\frac{k^{2}}{a^{2}}+M_{\mathrm{eff}}^{2}-i \frac{\left(a M_{\mathrm{eff}}\right)}{a}+\Delta(a)\right] X_{k}=0,
$$

where $\Delta(a) \equiv\left[\frac{1}{4}\left(\frac{\dot{a}}{a}\right)^{2}-\frac{1}{2}\left(\frac{\ddot{a}}{a}\right)\right]$. When $a \propto t^{n}$ such as in a matter or radiation dominated universe, $\Delta(a) \sim t^{-2}$ and can be neglected soon after inflation.

Using the mode equation and ansatz, we find

$$
\mathbf{u}_{k, \pm}(t)=a^{-1}\left[-i \dot{X}_{k}-i \frac{1}{2}\left(\frac{\dot{a}}{a}\right) X_{k}+\left(\gamma \cdot \mathbf{k}-M_{\mathrm{eff}}\right) X_{k}\right] R_{ \pm}(\mathbf{k})
$$

Taking the charge conjugate of $\mathbf{u}_{k, \pm}$, we find

$$
\mathbf{v}_{k, \pm}(t)=a^{-1}\left[i \dot{X}_{k}^{*}+i \frac{1}{2}\left(\frac{\dot{a}}{a}\right) X_{k}^{*}-\left(\gamma \cdot \mathbf{k}+M_{\mathrm{eff}}\right) X_{k}^{*}\right] \bar{R}_{ \pm}(\mathbf{k})
$$

where $\bar{R}_{ \pm}(\mathbf{k}) \equiv-i \gamma^{2} R_{ \pm}^{*}(\mathbf{k})$ is an eigenvector of helicity such that $\mathbf{k} \cdot \mathbf{\Sigma} \bar{R}_{ \pm}(\mathbf{k})= \pm 1$ and $\gamma^{0} \bar{R}_{ \pm}(\mathbf{k})=-1$.

The energy-momentum tensor is obtained from the ( $\psi$ and $\bar{\psi}$ symmetrized) matter action by variation with respect to the vierbein

$$
T^{\mu \nu}=\frac{i}{2}\left[\bar{\psi} \bar{\gamma}_{(\mu} \vec{D}_{\nu)} \psi-\bar{\psi} \overleftarrow{D}_{(\mu} \bar{\gamma}_{\nu)} \psi\right]
$$


and the Hamiltonian operator is

$$
\mathcal{H}_{D}=\int d^{3} x\left[i \psi^{\dagger} \dot{\psi}\right]
$$

In general, if this Hamiltonian is diagonal in the annihilation (and creation) operators $\hat{a}_{k, s}\left(\hat{a}_{k, s}^{\dagger}\right)$ and $\hat{b}_{k, s}\left(\hat{b}_{k, s}^{\dagger}\right)$ at $t=0$, it will not be for later times. This is the signature of particle creation due to the time dependent background. In order to determine the number of particles produced, we perform a Bogliubov transformation on the creation and annihilation operators so as to diagonalize the Hamiltonian at time $t$.

For the problem of particle creation quite often it is useful to represent the wave function in the adiabatic ( semi-classical, WKB) form

$$
X_{k}(t)=\alpha_{k} N_{+} e^{-i \int_{0}^{t} d t \Omega_{k}(t)}+\beta_{k} N_{-} e^{+i \int_{0}^{t} d t \Omega_{k}(t)},
$$

where $N_{ \pm}=\left(2 \Omega_{k}\left(\Omega_{k} \pm M_{\text {eff }}\right)\right)^{-1 / 2}$ and the coefficients $\alpha_{k}$ and $\beta_{k}$ correspond to the coefficients of the Bogliubov transformation.

Once the Bogliubov transformation is done, we may write the comoving number density of particles $n_{k}(t)=\left|\beta_{k}\right|^{2}$ in a given spin state through the solutions of the mode equation (9)

$$
n_{k}(t)=a\left(\frac{\Omega_{k}-M_{\mathrm{eff}}}{2 \Omega_{k}}\right)\left[\left|\dot{X}_{k}\right|^{2}+\Omega_{k}^{2}\left|X_{k}\right|^{2}-2 \Omega_{k} \operatorname{Im}\left(X_{k} \dot{X}_{k}^{*}\right)\right],
$$

where $M_{\text {eff }} \equiv\left(m_{\psi}+h \phi\right)$ as before and $\Omega_{k}^{2} \equiv \frac{k^{2}}{a^{2}}+M_{\text {eff }}^{2}$. The energy density in these particles is then

$$
\rho_{\psi}(t)=\frac{2}{a^{3}} \int \frac{d^{3} k}{(2 \pi)^{2}} \Omega_{k} n_{k}(t)=\frac{1}{a^{3} \pi} \int d k k^{2} \Omega_{k} n_{k}(t) .
$$

The normalization of the solutions $X_{k}(t)$ is such that $X_{k}\left(t \rightarrow 0^{-}\right)=N_{+} e^{-i \Omega_{k} t}$ and $n_{k}(0)=0$. Thus, we find $N_{+}=\left(2 \Omega_{k}\left(\Omega_{k}-M_{\text {eff }}\right)\right)^{-1 / 2}$. These are the so-called positive frequency initial conditions.

A comment about the regularization of fermion VEVs. In principle, fermionic VEVs like $\left\langle T^{\mu \nu}\right\rangle$ require regularization , which in the presence of background metrics and scalars is rather non-trivial, see e.g. [13] and references therein. We will consider only the processes of particle creation, ignoring vacuum polarization. The creation of particles, which corresponds to the imaginary part of the effective action, has no formal divergencies and does not require regularization. Assuming the particle creation process dominates over vacuum polarization, we will not consider the issues of regularization.

\section{FERMIONIC PREHEATING WITHOUT EXPANSION OF THE UNIVERSE}

It is convenient to begin the investigation of fermionic preheating due to an oscillating scalar field with a simplified setting neglecting the expansion of the universe. This setting may have not only methodological advantages. Indeed, whenever the frequency of $\phi$ oscillations is much greater than the rate of cosmic expansion $H$, it is sensible to neglect the time dependence of the scale factor in solving the mode equation (9). This can be the case, for example, when spontaneous symmetry breaking occurs rapidly leaving the $\phi$-field oscillating about a new minimum where the effective mass $m_{\phi}$ happens to be much larger than the Hubble parameter at the time of the transition. One such example is hybrid inflation scenarios which end with TeV scale energy densities. Another example is the inflationary model with the potential $\lambda \phi^{4}$. This theory possesses conformal properties: at the stage of inflaton oscillations equations for the fields by means of conformal transformations can be reduced to the equations in Minkowskii space-time, see e.g. [5].

In this paper we will mostly use a chaotic inflationary model with quadratic potential $V(\phi)=\frac{1}{2} m_{\phi}^{2} \phi^{2}$. If we make the replacement $a=1$ in all the formulas of Section 2, all the effects of expansion will be removed. Background oscillations take the form of harmonic oscillations $\phi(t)=\phi_{0} f(t)$ with $f(t)=\cos \left(m_{\phi} t\right)$ and $\phi_{0}$ the time independent amplitude. It is convenient to define a new, dimensionless time variable $\tau \equiv m_{\phi} t$. With this change of variables, the mode equation (9) may be written

$$
X_{k}^{\prime \prime}+\left[\kappa^{2}+(\tilde{m}+\sqrt{q} f)^{2}-i \sqrt{q} f^{\prime}\right] X_{k}=0,
$$

where we have introduced the dimensionless momentum $\kappa \equiv \frac{k}{m_{\phi}}$, the dimensionless fermion mass $\tilde{m} \equiv \frac{m_{\psi}}{m_{\phi}}$, and the resonance parameter $q \equiv \frac{h^{2} \phi_{0}^{2}}{m_{\phi}^{2}}$. These three parameters completely determine the strength of the effect. In fact, this 
form of the mode equation is valid not only for harmonic background oscillations, but for generic $\phi$ oscillations in a general potential. For this, we identify $m_{\phi}$ with the frequency of oscillation, $\phi_{o}$ with its amplitude, and $f(\tau)$ with the periodic background oscillations normalized to unit amplitude. Note that the frequency will be amplitude-dependent for a general, non-quadratic potential.

\section{A. Parametric Excitation of Fermions}

If individual inflatons at rest are decaying into light fermions in a process $\phi \rightarrow \bar{\psi} \psi$, perturbative calculations give the rate of decay $\Gamma_{\phi \rightarrow \psi \psi} \simeq \frac{h^{2} m}{8 \pi}$ and the spectrum of created fermions is sharply peaked around $m / 2$ with the width $\Gamma_{\phi \rightarrow \psi \psi}^{-1}$. In Figs. 14 2, however, we plot the time-dependence and spectrum of occupation number for fermions created from the coherently oscillating inflaton field, as follows from numerical solution of Eq. (17) and (15).

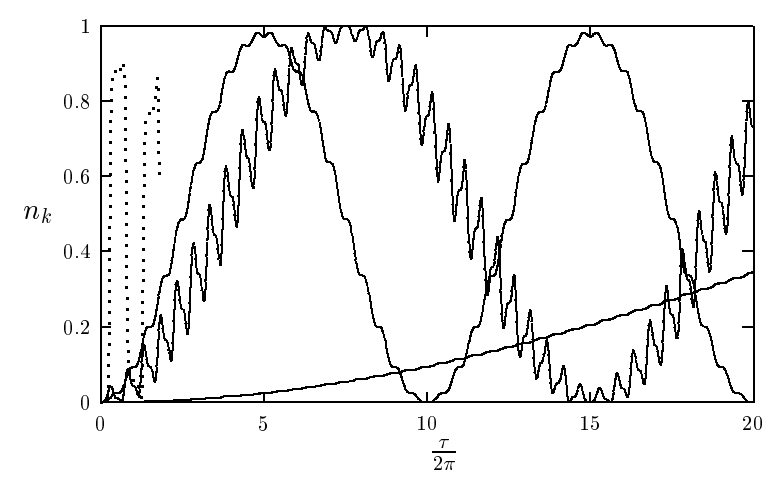

FIG. 1. The occupation number $n_{k}$ of fermions in $m_{\phi}^{2} \phi^{2}$-theory as function of time $\frac{\tau}{2 \pi}$ for a range of $(q ; \kappa)$ : dotted curve for $(1.0 ; 0.05)$, bold curve for $\left(10^{-4} ; 0.5\right)$, smooth sine-like curve for $\left(10^{-2} ; 0.5\right)$, wiggly sine-like curve for $(1 ; 1.3)$.

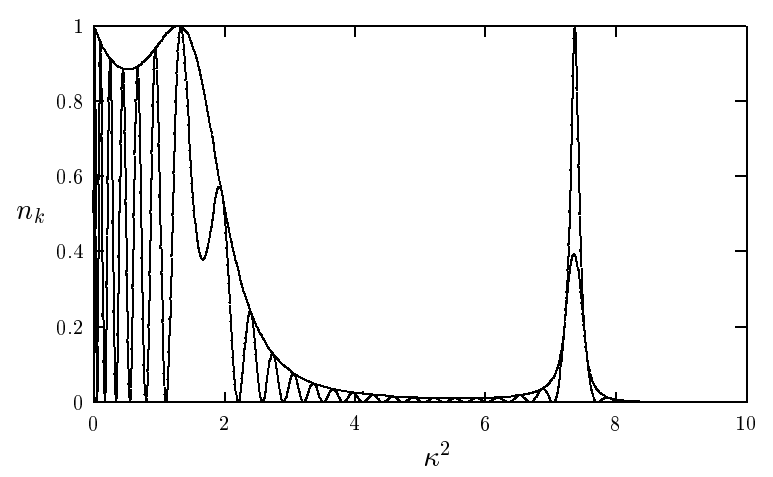

FIG. 2. The comoving occupation number of fermions in $m_{\phi}^{2} \phi^{2}$-inflation as a function of $\kappa^{2}$ after 10 inflaton oscillations for resonance parameter $q=10$. Also shown is the envelope function $F_{k}$ defined by equation (21).

The spectrum and time evolution are drastically different from what is expected from the perturbative calculations. We therefore can talk about a specific phenomena, the parametric excitation of fermions interacting with coherent background oscillations.

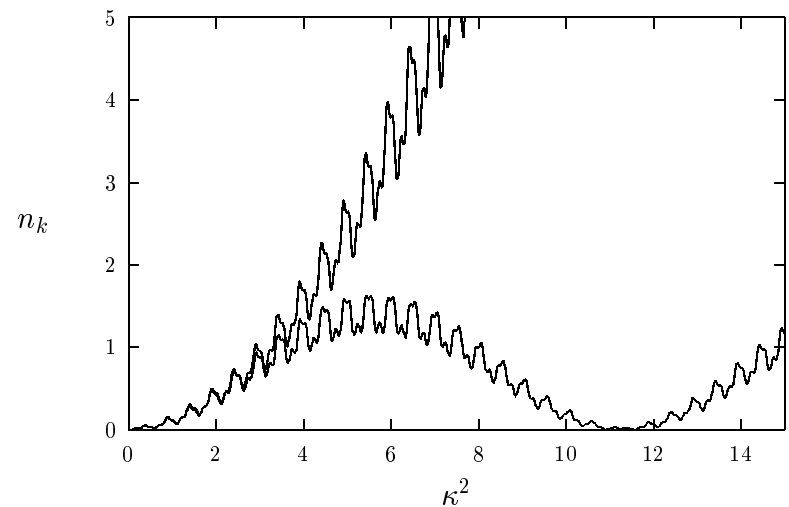

FIG. 3. Time dependence of the occupation number in $\lambda \phi^{4}$-theory for $q=3$ for two different modes: one is just inside the resonance band which grows like $\sinh \left(\mu_{k} t\right) \sim e^{\mu_{k} \tau}$ and another outside which oscillates like $\sin \left(\mu_{k} t\right)$.

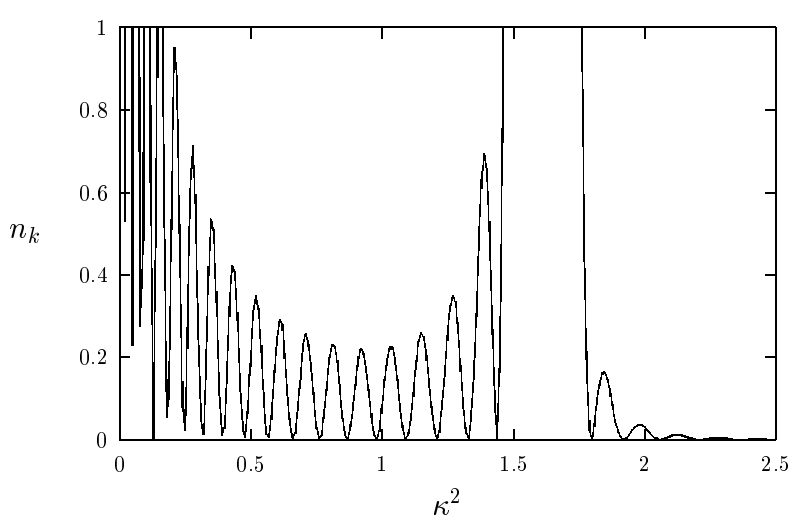

FIG. 4. Bosonic occupation number after 10 background oscillations for $\lambda \phi^{4}$-theory when $q=\frac{g^{2}}{\lambda}=3$. A stripe with sharp edges corresponds to the $e^{\mu_{k} \tau}$ instability.

It is instructive to compare the spectrum and evolution of fermionic occupation number with those of bosonic occupation number. The mode function of a quantum bose scalar field $\hat{\chi}$ coupling as $g^{2} \chi^{2} \phi^{2}$ with oscillating inflaton 
obeys the bosonic oscillator-like equation

$$
X_{b, k}^{\prime \prime}+\left[\kappa^{2}+\tilde{m}_{b}^{2}+q_{b} f^{2}\right] X_{b, k}=0
$$

where $q_{b}=\frac{g^{2} \phi_{0}}{m^{2}}, \tilde{m}_{b}=\frac{m_{\chi}}{m}$. In Figs. 国 and 3 we plot the spectrum and time evolution of bosonic occupation number calculated from bosonic oscillator-like equations (18). We use the familiar model of bosonic resonance due to the self-interaction in $\lambda \phi^{4}$ inflation, which corresponds to $g^{2}=3 \lambda, q_{b}=3, m_{b}=0, f(\tau)$ is given by oscillations in $\lambda \phi^{4}$ theory.

In the bosonic case, there are distinct resonance bands in which bosonic modes are exponentially unstable, $n_{k}^{b}(t) \sim$ $e^{\mu_{k} \tau}$. Particle creation takes place outside of the resonant bands as well, although there the occupation number is bounded and oscillates periodically. In the fermionic case $n_{k} \leq 1$ is always bounded by Pauli blocking and oscillating periodically with time. The occupation number in both cases is changing in time. It is therefore convenient to introduce an envelope function $F_{k}$ of the particle spectrum [5], which corresponds to $n_{k}$ averaged over short-time intervals (order of the background oscillation period). A bosonic envelope function cannot be defined for the resonant bands (where it is $e^{\mu_{k} \tau}$.) This zone corresponds to the gap between almost vertical lines in Fig. 1 . The fermionic envelope function $F_{k}$, on the contrary, can be defined everywhere. Although it is always bounded by unity, their structure is reminiscent of the resonant-band structure: for some range of $k$, the 'resonant' band, $n_{k}$ is close to unity, while in other ranges, the stable bands, it is significantly smaller if not zero. In [12] different levels of $F_{k}$ were plotted on the parameter plane $(\kappa, q)$, revealing a structure which reminiscent of the stability/instability chart of the bosonic parametric resonance. One of the most important results is that fermionic parametric excitations occurs very quickly, within about ten(s) background oscillations. Interestingly, fermionic "resonant bands" are excited the last, while non-resonant intervals fill first.

Comparison of bosonic and fermionic parametric excitations is useful to understand some features of bosonic preheating. As we have seen, there is production of bosons outside of the resonance band. If we take into account the expansion of the universe, in the most interesting case of large $q_{b}$, the difference between resonant and non-resonant excitations of bosons will be erased, and the regime of stochastic resonant production of bosons will be settled down [2].

\section{B. Some Generic Analytic Results}

Dynamics of the Fermi field coupling to the background homogeneous scalar can be revealed with the second-order oscillator-like equation (17) for the mode function $X_{k}(t)$. For periodic background oscillations $f(t)=f(t+T), T$ is a period, some generic analytic results were derived a long time ago [10] in the context of particle creation in a periodic external electromagnetic field. In particular, the occupation number of created particles at instances $t=N_{s} T$, i.e. exactly after $N_{s}$ background oscillations, is given by expression [10]

$$
n_{k}\left(N_{s} T\right)=\frac{k^{2}}{2 \Omega_{k}^{2}} \frac{\sin ^{2} N_{s} d_{k}}{\sin ^{2} d_{k}}\left(\operatorname{Im} X_{k}^{(1)}(T)\right)^{2}
$$

where $\cos d_{k}=\operatorname{Re} X_{k}^{(1)}(T)$. To derive this result, one introduces two fundamental solutions of Eq. (17), $X_{k}^{(1)}(t)$ and $X_{k}^{(2)}(t)$; such that initially $X_{k}^{(1)}(0)=1, \dot{X}_{k}^{(1)}(0)=0$ and $X_{k}^{(2)}(0)=0, \dot{X}_{k}^{(2)}(0)=1$. Expression (19) involves only the value of the first fundamental solution $X_{k}^{(1)}(T)$ exactly after the first oscillation. It says that the occupation number of created particles after $N_{s}$ background oscillations is modulated with a certain frequency $\nu_{k}$ (which, as we will see, does not coincide with $d_{k}$ ). However, practical application of the generic formula (19) is rather limited, because it does not address the full time evolution of $n_{k}(t)$, and cannot strictly determine a period of modulation $\pi / \nu_{k}$.

To get an idea of how the occupation number of created particles $n_{k}(t)$ evolves with time, again let us look at Fig. 1 and further at Fig. 7 for different values of the parameters $\kappa$ and $q$. For small and moderate $q$ (but not too small $\kappa)$ the occupation number exhibits high frequency (period $<\frac{T}{2}$ ) oscillations which are modulated by a long period behavior. For large $q$ number of fermions jumps in a step-like manner at instances when effective mass of the fermi field crosses zero, superposed by very high frequency oscillations around almost constant values, as depicted in Fig. 7. These jumps are modulated with a frequency $\nu_{k} / 2$ : steps in the first half of the cycle up are accumulated until $n_{k}(t)$ reaches its maximum $F_{k}$, and then steps down to zero in the second half of the cycle.

However, this picture of high frequency features superimposed over long-period modulation is not universal. For small $\kappa$, or for one of the most interesting cases of $q \gg 1$ and moderate $\kappa$, the occupation number of fermions jumps between zero and one within time interval much shorter than period of background oscillations, as depicted by the 
dotted curve in Fig. 1. There are interesting situations when fermions are created in an instant (single kick) process, where formula (19) is not applicable. Therefore, for different ranges of parameters we will shall develop different approaches.

\section{Semi-Analytic Theory for Averaged Occupation Number}

Numerical curves for the mode functions suggests splitting of the time evolution into higher frequency features with the time-scale comparable or less than the period of background oscillations $\leq T$, and low-frequency modulations with the period $\pi / \nu_{k}$ greater than $T$. In this case we can utilize generic result (19). However, it is convenient to use not the values $n_{k}\left(N_{s} T\right)$, but rather the smoothed occupation number $\bar{n}_{k}(t)$ which is $n_{k}(t)$ averaged over high frequency oscillations, $\bar{n}_{k}(\tau)=\frac{1}{T} \int_{\tau}^{(\tau+T)} d \tau n_{k}(\tau)$. Then we can write the smoothed occupation number of fermions in a factorized form

$$
\bar{n}_{k}(\tau)=F_{k} \sin ^{2} \nu_{k} \tau
$$

where we introduce an envelope function $F_{k}$. The average occupation number of fermions evolves periodically with time. The spectrum of $\bar{n}_{k}$ can be characterized by the envelope function $F_{k}$ and the period of modulation $\frac{\pi}{\nu_{k}}$ which depends also on the parameter $q$.

Now we will utilize the result (19). We found that the envelope function can be extrapolated by the factor in the front of $\sin ^{2} N_{s} d_{k}$ in (19) and given by the expression

$$
F_{k}=\frac{1}{\sin ^{2} \nu_{k} T} \frac{\kappa^{2}}{2 \Omega_{k}^{2}}\left(\operatorname{Im} X_{k}^{(1)}(T)\right)^{2} .
$$

Next is to determine the frequency $\nu_{k}$ of the $\bar{n}_{k}$ modulations. The value $d_{k}$ defined after (19) cannot be the right answer, because it would incorrectly predict that the peaks of $F_{k}$ are filled up first, while actually they are filled last. We tried the combination $\nu_{k}=\pi / 2-d_{k}$, because it corresponds to correct order of saturation of $F_{k}$, and it occurs to work well. Therefore, the modulation frequency $\nu_{k}$ is given by the relation $\cos \nu_{k} T=-R e X_{k}^{(1)}(T)$. Thus, to find $F_{k}$ and $\nu_{k}$, one need only calculate the complex value $X_{k}^{(1)}(T)$ after a single background oscillation, instead of performing a full numerical integration of Eq. (17).

We calculated $X_{k}^{(1)}(T)$ numerically for $\frac{1}{2} m^{2} \phi^{2}$ background model and constructed the envelope function $F_{k}$ plotted in Fig. 5 (similar graph was plotted in [5] for $\frac{1}{4} \lambda \phi^{4}$ model). In Fig. 2 2 we show, using (21]), how the fermionic resonance bands are filled after 10 background oscillations.

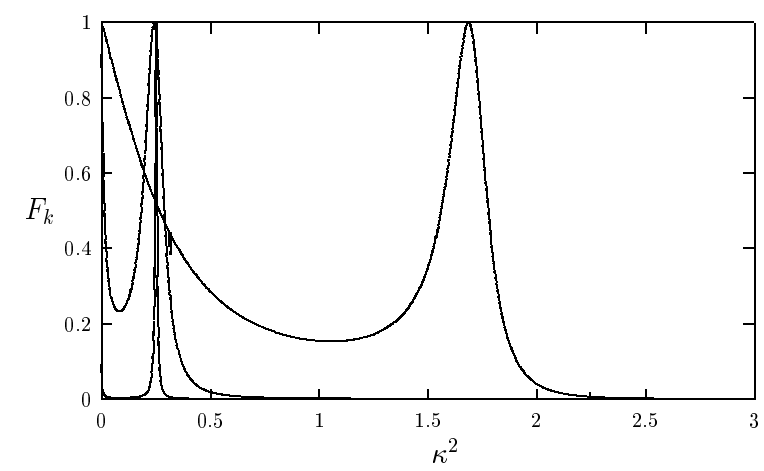

FIG. 5. The envelope function $F_{k}$ for the amplitude of occupation number oscillations in $m_{\phi}^{2} \phi^{2}$-theory. Values for $q=10^{-4}, 10^{-2}$ and 1 are shown, from narrowest to broadest, respectively.

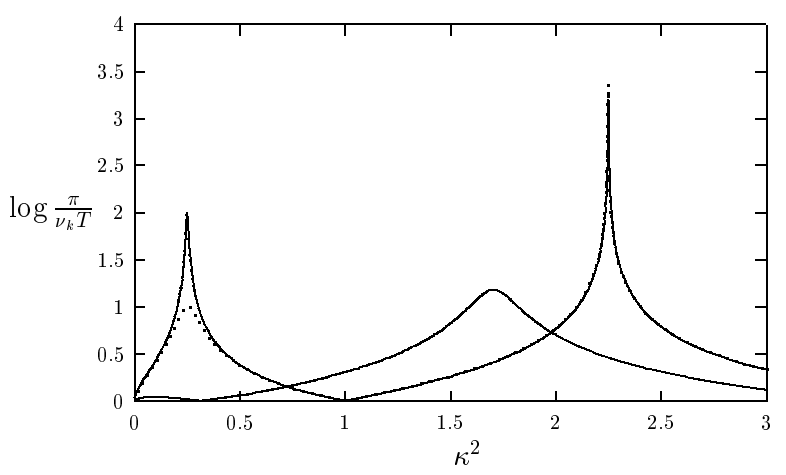

FIG. 6. The log of the modulation period in $m_{\phi}^{2} \phi^{2}$-theory, where $q=1$ corresponds to the middle curve, $q=10^{-2}$ corresponds to the dotted curve, and $q=10^{-4}$ corresponds to the remaining.

The function $\nu_{k}$ gives us the time scale for fermion excitation. In Fig. 6 we plot the period of modulation $\frac{\pi}{\nu_{k}}$ as a function of $k$. This function is peaked where $F_{k}$ is peaked, i.e. the peaks of the resonance curve are the last to fill. 


\section{Method of Successive Scatterings for Fermions}

It turns out that for large values of the parameter $q$ we can significantly advanced in calculations of $n_{k}(t)$ beyond the results of Section (III ). One can expect that $q$ may be much greater that one. Indeed, $q=\frac{h^{2} \phi_{0}^{2}}{m^{2}}$. In the context of chaotic inflation with the potential $V(\phi)=\frac{1}{2} m^{2} \phi^{2}$ it follows from the theory of cosmological perturbations that $\frac{\phi_{0}^{2}}{m^{2}} \simeq 10^{12}$. On the other hand one can admit that Yukawa coupling can be $h \gg 10^{-6}$, which provides $q \gg 1$.

We will generalize for fermions the method of parabolic scatterings introduced for the bosonic resonance in reference [2]. The method is based on the observation that, for $q \gg 1$, change of the particle number occurs only during a short time interval $\tau_{*}$ near the zeros of the effective mass of the particles. This occurs because equation (15) for the number of particles in terms of the mode functions is an adiabatic invariant of the mode equation.

For large $q$, the adiabaticity condition $\Omega_{k}^{\prime}<\Omega_{k}^{2}$ is violated only near the times $\tau_{*}$ when the effective mass vanishes. This leads to a step-like evolution in the number of fermions. This is illustrated in figure (7) which shows the evolution of $n_{k}(\tau)$ and $M_{\text {eff }}(\tau)=h \phi(\tau)+m_{\psi}$

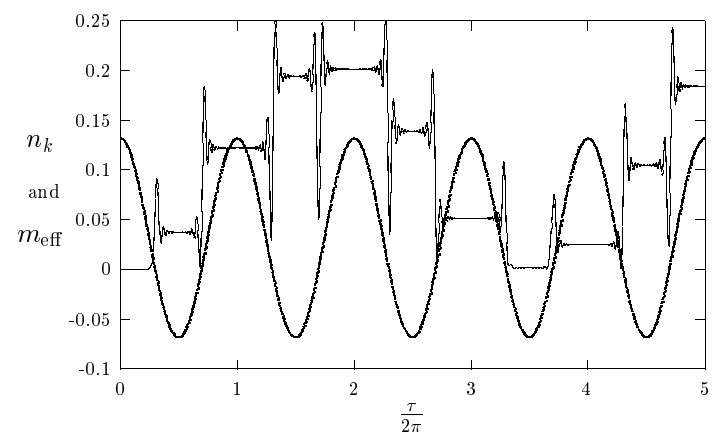

FIG. 7. $n_{k}$ and $M_{\mathrm{eff}}(\tau)=h \phi(\tau)+m_{\psi}$ for $q=100$ and $\kappa=\sqrt{q} \approx 31.6$.

Near the times $\tau_{*}$, we may approximate the effective mass in equation $(17)$ by $(\tilde{m}+\sqrt{q} f)=\frac{1}{m_{\phi}}\left(m_{\psi}+h \phi(\tau)\right) \approx$ $\frac{h}{m_{\phi}} \phi\left(\tau_{*}\right)^{\prime}\left(\tau-\tau_{*}\right)+O\left(\left(\tau-\tau_{*}\right)^{2}\right)$. We may also write this as

$$
(\tilde{m}+\sqrt{q} f) \approx \sqrt{q} f^{\prime}\left(\tau_{*}\right)\left(\tau-\tau_{*}\right)= \pm\left(q-\tilde{m}^{2}\right)\left(\tau-\tau_{*}\right),
$$

where the sign on the left hand side depends on whether $f(\tau)$ is increasing $(+)$ or decreasing $(-)$ at $\tau_{*}$. Thus, in the neighborhood of $\tau_{*}$, the mode equation becomes

$$
X_{k}^{\prime \prime}+\left[\kappa^{2}+\left(q-\tilde{m}^{2}\right)^{2}\left(\tau-\tau_{*}\right)^{2}-i \operatorname{sgn}\left(f^{\prime}\right) \sqrt{q-\tilde{m}^{2}}\right] X_{k}=0
$$

which is a Schroedinger equation for scattering off a negative parabolic potential centered at $\tau_{*}$.

The method of parabolic scattering uses the exact solution of equation (23) to provide a connection formula between the adiabatic approximations of the full mode equation on either side of $\tau_{*}$. Suppose the $j$-th zero of $M_{\text {eff }}$ occurs at time $\tau_{j}$. For times between $\tau_{j-1}<\tau<\tau_{j}$, the general solution of the mode equation (17) takes the adiabatic (or semi-classical) form

$$
X_{k}^{j}(\tau)=\alpha_{k}^{j} N_{+} e^{-i \int_{0}^{\tau} d \tau \Omega_{k}(\tau)}+\beta_{k}^{j} N_{-} e^{+i \int_{0}^{\tau} d \tau \Omega_{k}(\tau)},
$$

where the coefficients $\alpha_{k}^{j}$ and $\beta_{k}^{j}$ are constant for $\tau_{j-1}<\tau<\tau_{j}$. After the scattering at $\tau_{j}, X_{k}(t)$, within the interval $\tau_{j}<\tau<\tau_{j+1}$, again has the adiabatic form

$$
X_{k}^{j+1}(\tau)=\alpha_{k}^{j+1} N_{+} e^{-i \int_{0}^{\tau} d \tau \Omega_{k}(\tau)}+\beta_{k}^{j+1} N_{-} e^{+i \int_{0}^{\tau} d \tau \Omega_{k}(\tau)},
$$

with new coefficients $\alpha_{k}^{j+1}$ and $\beta_{k}^{j+1}$ that are constant for $\tau_{j}<\tau<\tau_{j+1}$. At $\tau=0$, our vacuum positive frequency condition requires $\alpha_{k}^{1}=1$ and $\beta_{k}^{1}=0$. Particle creation occurs when, after scattering at the times $\tau_{j}$, the initial positive 
frequency wave acquires a negative frequency part. The number density of produced particles with momentum $k$ is $n_{k}^{j}=\left|\beta_{k}^{j}\right|^{2}$ for times $\tau_{j}<\tau<\tau_{j+1}$. Furthermore, normalization requires $\left|\alpha_{k}^{j}\right|^{2}+\left|\beta_{k}^{j}\right|^{2}=1$ for all $j$.

The important observation is that the outgoing amplitudes $\left(\alpha_{k}^{j+1}, \beta_{k}^{j+1}\right)$ can be expressed through the incoming amplitudes $\left(\alpha_{k}^{j}, \beta_{k}^{j}\right)$ by means of the reflection $R_{k}$ and transmission $D_{k}$ amplitudes for scattering at $t_{j}$ :

$$
\left(\begin{array}{c}
\alpha_{k}^{j+1} e^{-i \theta_{k}^{j}} \\
\beta_{k}^{j+1} e^{+i \theta_{k}^{j}}
\end{array}\right)=\left(\begin{array}{cc}
\frac{1}{D_{k}} & \frac{R_{k}^{*}}{D_{k}^{*}} \\
\frac{R_{k}}{D_{k}} & \frac{1}{D_{k}^{*}}
\end{array}\right)\left(\begin{array}{c}
\alpha_{k}^{j} e^{-i \theta_{k}^{j}} \\
\beta_{k}^{j} e^{+i \theta_{k}^{j}}
\end{array}\right)
$$

Here $\theta_{k}^{j}=\int_{0}^{\tau_{j}} d \tau \Omega(\tau)$ is the phase accumulated by the moment $\tau_{j}$.

If we let $x=\left(q-\tilde{m}^{2}\right)^{1 / 4} \tau$, equation (23) may be written

$$
\frac{d^{2} X_{k}}{d x^{2}}+\left[\Lambda_{k}^{2}-i(-1)^{j}+x^{2}\right] X_{k}=0
$$

where the parameter

$$
\Lambda_{k}^{2} \equiv \frac{\kappa^{2}}{\left|h f^{\prime}\left(\tau_{*}\right)\right|}=\frac{\kappa^{2}}{\sqrt{q-\tilde{m}^{2}}} .
$$

A general analytic solution of equation (27) is a linear combination of the parabolic cylinder functions $W\left(-\frac{\left(\Lambda_{k}^{2}-i(-1)^{j}\right)}{2} ; \pm \sqrt{2} x\right)$. The reflection $R_{k}$ and transmission $D_{k}$ amplitudes for scattering on the parabolic potential can be found from the asymptotic forms of these analytic solutions:

$$
R_{k}=-\frac{i e^{i \varphi_{k}}}{\sqrt{1-e^{\pi \Lambda_{k}^{2}}}}, D_{k}=\frac{e^{-i \varphi_{k}}}{\sqrt{1-e^{-\pi \Lambda_{k}^{2}}}},
$$

where the angle $\varphi_{k}$ is $\varphi_{k}=\arg \Gamma\left(\frac{1+i \Lambda_{k}^{2}}{2}\right)+\frac{\Lambda_{k}^{2}}{2}\left(1+\ln \frac{2}{\Lambda_{k}^{2}}\right)$. Note that the angle $\varphi$ depends on the momentum $k$. Substituting (29) into (26), we can determine the change in induced in the $\alpha_{k}$ and $\beta_{k}$ coefficients by a single parabolic scattering in terms of the parameters of the parabolic potential and the phase $\theta_{k}^{j}$ only. Specifically, we find

$$
\left(\begin{array}{c}
\alpha_{k}^{j+1} \\
\beta_{k}^{j+1}
\end{array}\right)=\left(\begin{array}{cc}
\sqrt{1-e^{-\pi \Lambda_{k}^{2}}} e^{i \varphi_{k}} & -(-1)^{j} e^{-\frac{\pi}{2} \Lambda_{k}^{2}+2 i \theta_{k}^{j}} \\
(-1)^{j} e^{-\frac{\pi}{2} \Lambda_{k}^{2}-2 i \theta_{k}^{j}} & \sqrt{1-e^{-\pi \Lambda_{k}^{2}}} e^{-i \varphi_{k}}
\end{array}\right)\left(\begin{array}{c}
\alpha_{k}^{j} \\
\beta_{k}^{j}
\end{array}\right) .
$$

It is now a simple matter to find the change in particle number after one scattering. From the normalization of $\alpha_{k}$ and $\beta_{k}$ we have the relation

$$
\left(\begin{array}{cc}
\alpha_{k}^{* j+1} & \beta_{k}^{* j+1}
\end{array}\right)\left(\begin{array}{cc}
1 & 0 \\
0 & -1
\end{array}\right)\left(\begin{array}{c}
\alpha_{k}^{j+1} \\
\beta_{k}^{j+1}
\end{array}\right)=\left|\alpha_{k}\right|^{2}-\left|\beta_{k}\right|^{2}=1-2 n_{k}^{j+1},
$$

which, when applied to equation (30) gives the desired result

$$
\begin{aligned}
n_{k}^{j+1} & =e^{-\pi \Lambda_{k}^{2}}+\left(1-2 e^{-\pi \Lambda_{k}^{2}}\right) n_{k}^{j} \\
& -2(-1)^{j} e^{-\frac{\pi}{2} \Lambda_{k}^{2}} \sqrt{1-e^{-\pi \Lambda_{k}^{2}}} \sqrt{n_{k}^{j}\left(1-n_{k}^{j}\right)} \sin \theta_{t o t}^{j},
\end{aligned}
$$

where the phase $\theta_{\text {tot }}^{j}=2 \theta_{k}^{j}-\varphi_{k}+\arg \beta_{k}^{j}-\arg \alpha_{k}^{j}$. Let us discuss this formula, which is the main result of our paper. If fermions are light, $m_{\psi}=0$, their occupations number is changing with time only when background field crosses zeros. Without expansion of the universe, the phases $\theta$ accumulated between successive zeros are equal. In this case one can try to proceed to find the solution of the matrix equation (26), as it was done for bosons [2]. However, for bosons that was needed to find the stability/instability bands, which is not so interesting for fermions. In the case of massive fermions time intervals between successive zeros of $M_{e f f}$ are not equal, and problem of finding matrix solution of eq. (26) became even more complicated. However, in the most interesting case of expanding universe the phases between successive zeros of $M_{\text {eff }}$ became random (see [2] for details). Then we can just put $\theta_{\text {tot }}$ as a random phase and use formula (32) as it is. 


\section{PARAMETRIC EXCITATION OF FERMIONS WITH EXPANSION OF THE UNIVERSE}

To address the problem of fermionic preheating after $m^{2} \phi^{2}$ chaotic inflation, we must now deal with the full mode equation (9), which no longer has a periodic time dependence in the complex frequency. Nevertheless, it is still convenient to work with the form (17) in the time variable $\tau=m_{\phi} t$ where the parameters $q$ and $\kappa^{2}$ are now understood to be time dependent:

$$
X_{k}^{\prime \prime}+\left[\frac{\kappa^{2}}{a^{2}}+M_{\mathrm{eff}}^{2}-i \frac{\left(a M_{\mathrm{eff}}\right)^{\prime}}{a}+\Delta(a)\right] X_{k}=0
$$

Check where' stands for the derivative in respect with $\tau, \kappa$ is a comoving momentum scaled in units of $m_{\phi}$. There is often used another from of the fermionic mode equation written in terms of conformal time $\eta=\int d t / a$ and mode function $Y_{k}=a^{-3 / 2} \chi_{k}$

$$
\partial_{\eta}^{2} Y_{k}+\left[\kappa^{2}+M_{\mathrm{eff}}^{2}-i \partial_{\eta}\left(a M_{\mathrm{eff}}\right)\right] Y_{k}=0 .
$$

This form of equation is useful, for instance, in conformal theory $V(\phi)=\lambda \phi^{4}$ when the problem can be reduced to the problem in Minkowski space-time [5]. In this case background field is oscillating periodically in respect with time $\eta$. In the case of quadratic inflaton potential background field is oscillating with the constant period in terms of physical time $t$ (or $\tau$ ). We therefore will use mode equation (33). The parameter $q$ is now understood to be time dependent. Specifically, we have $q(\tau) \equiv \frac{h^{2} \Phi^{2}(\tau)}{m_{\phi}^{2}}$, scaled physical momentum $p \equiv \frac{1}{a} \frac{k}{m_{\phi}}$ Here, $\Phi(\tau)$ is the time dependent amplitude of inflaton oscillations. As is well known (c.f. [2]), oscillations of $\phi$ in this model quickly approach the asymptotic solution $\phi(\tau)=\Phi(\tau) \cos (\tau), \Phi(\tau) \approx \frac{\phi_{0}}{a^{3 / 2}} \sim \frac{M_{\mathrm{pl}}}{\sqrt{3 \pi}(1+\tau)}$ where time is measured from the start of inflaton oscillations, $\phi_{0} \approx \frac{M_{\mathrm{pl}}}{\sqrt{3 \pi}}$, and the scale factor averaged over several oscillations behaves as in a matter dominated universe: $a(\tau)=(1+\tau)^{2 / 3}$.

\section{A. Stochastic Parametric Excitations of Fermions}

Let us first consider the case of light fermions with a large initial resonance parameter: $h \phi_{0} \gg m_{\phi} \gg m_{\psi}$. Fig. (8) shows a numerical solution of equation (17) in the absence of expansion with a resonance paramter $q=10^{6}$. As expected from for such a large $q$ parameter, we see a step-like change in occupation number with periodic modulation.

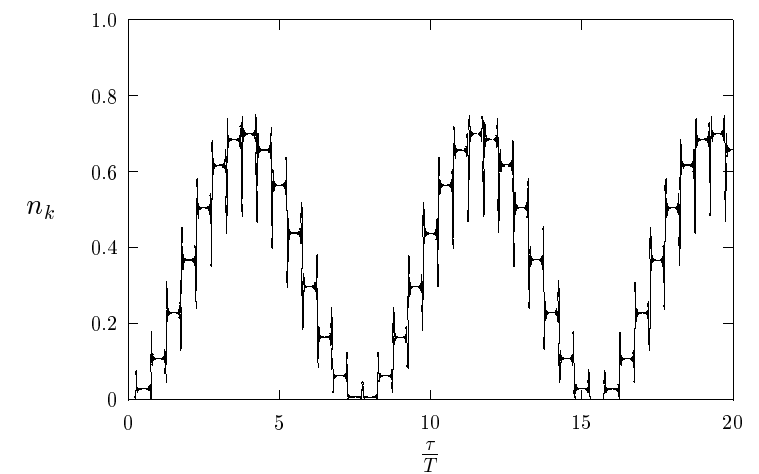

FIG. 8. The occupation number $n_{k}$ of fermions in $m_{\phi}^{2} \phi^{2}$-theory without expansion. Here $q=10^{6}$ and the particular mode is $\kappa^{2} \approx \sqrt{q}$.

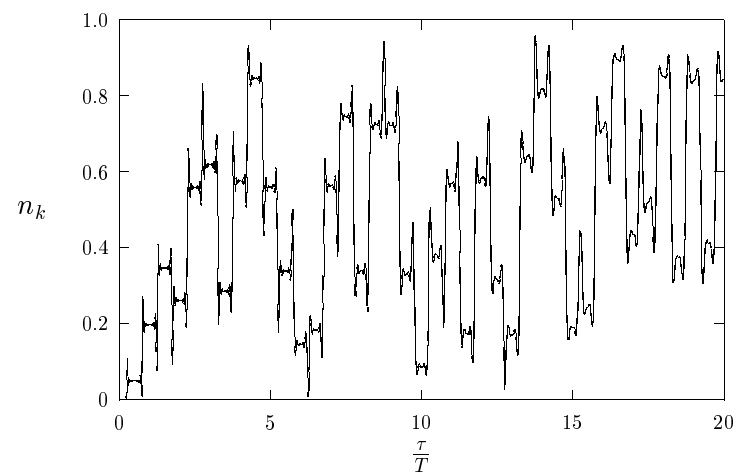

FIG. 9. The occupation number $n_{k}$ of fermions in $m_{\phi}^{2} \phi^{2}$-theory with expansion taken into account. Here the initial resonance parameter is $q_{0}=10^{6}$ and the mode is $\kappa_{0}^{2} \approx \sqrt{q}$.

In Fig. (9), we show a numerical solution to the equation (33) in the presence of expansion with the initial parameters $q_{0}=10^{6}$. Here, the comoving number density of particles is plotted. We see that, as for the non-expanding case, evolution between the zeros of the effective mass (oe equally inflaton field), $M_{\mathrm{eff}} \approx \sqrt{q(\tau)} \cos (\tau)$, is adiabatic. We can thus apply the results of Section III,D in particular the analytic formula (32), mutatis mutandis. The Pauli principle is obviously still obeyed and the typical step in particle number is still suppressed by a factor $e^{-\pi \Lambda_{k}}$ which is now time dependent. The most important qualitative change is that the accumulated phase $\theta_{\text {tot }}$ is now 
uncorrelated between successive zeros of the effective mass (inflaton field). This occurs because the effective frequency $\Omega_{k}=\sqrt{p^{2}+q^{2}(\tau) \cos ^{2} \tau}$ is no longer periodic and the accumulated phase $\theta=\int_{j}^{j+1} \Omega_{k} \approx \frac{2 h \Phi(\tau)}{m}+O\left(k^{2}\right)$ changes substantially in magnitude within one inflaton oscillation, $\delta \theta_{k} \simeq \frac{\sqrt{q}}{2 N_{s}^{2}}$, after the $N_{s}$-th oscillation [2]. The result is that the $\sin \left(\theta_{\text {tot }}\right)$ term in (32) becomes a random variable. As is readily apparent in Fig. (9), this destroys the periodic modulation of $n_{k}$ and the parametric excitation of fermions becomes stochastic, as anticipated in [5].

Once the periodic modulation of $n_{k}$ is destroyed, the construction of Section III,C is no longer valid: the occupation number cannot be characterized by an amplitude and period. In fact, the spectrum of created particles is even simpler. Stochastic excitation allows a given comoving mode $\kappa$ to obtain any amplitude in the range $0 \leq n_{k} \leq 1$ if there are a sufficient number of parabolic scatterings, $N_{s}$. This gives us the picture of stochastically filling a (Fermi) sphere in the momentum space. Numerical calculations confirm this picture, see Fig. 10. Let us find its radius $\kappa_{s}$. A comoving mode will be excited if $\Lambda_{k}(\tau) \leq 1 / \pi$. Since $\Lambda_{k}(\tau)=\frac{\kappa^{2}}{a^{2} \sqrt{q(\tau)}}$, we have

$$
\frac{\kappa_{s}^{2}}{a^{2} \sqrt{q(\tau)}}=a^{-1 / 2} \frac{\kappa_{s}^{2}}{\sqrt{q_{0}}} \leq 1 / \pi
$$

Therefore for the light fermions, comoving radius of excited modes is increasing with time as $\kappa_{s} \sim a^{1 / 4}$. The radius of the sphere is scaled as $\kappa_{s} \sim q_{0}^{1 / 4} a^{1 / 4}$.

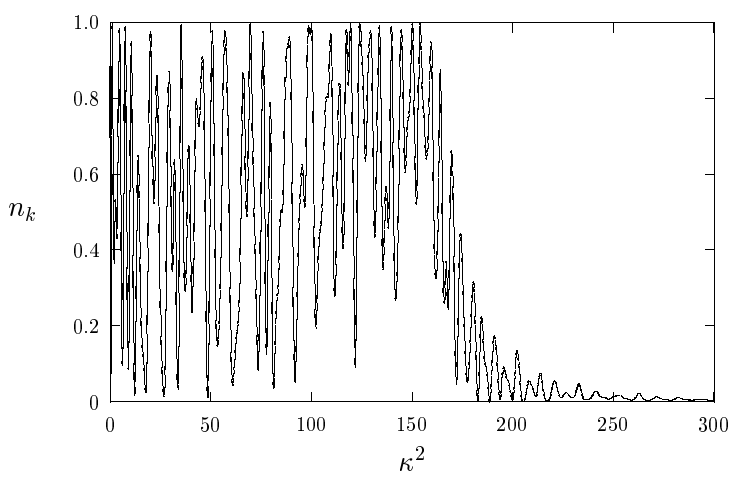

FIG. 10. The comoving occupation number of fermions in $m_{\phi}^{2} \phi^{2}$-inflation after 50 inflaton oscillations for initial resonance parameter $q_{o}=10^{3}$. Expansion destroys the details of the resonance band and leads to a fermi-sphere of width $q^{1 / 4}$ which grows like $a^{1 / 4}$ while $q(\tau)>1$.

Due to the expansion, the amplitude $\Phi$ is decreasing and the parameter $q(\tau)$ drops. At some moment the Fermi sphere will quit expansing. Once $q(\tau)$ is order of unity, the excitation is no longer strong, and redshifts of fermion modes will be fast enough to prevent from parametric excitation. Eventually fermions will be produced in the perturbative regime. If fermionic mass is non-zero, the perturbative decay goes unless $2 m_{\psi}>m_{\phi}$.

\section{B. Analytic Results for Production of Supermassive Fermions}

One of the most significant differences between bosonic and fermionic parametric amplification is the possibility to create superheavy fermions from much liter inflatons, if inflatons are oscillating coherently. As we seen, for large $q$, massive fermions are created not when inflaton field itself crosses zero, but when the combination $M_{e f f}=h \phi+m_{\psi}$ crosses zero. This is because at those instances even very heavy fermions are effectively massless, because their bare mass is "compensated" by large value of $h \phi$, if $\phi$ can have a large amplitude. This is exactly the case when $\phi$ is the inflaton field. For instance, in the chaotic inflationary scenario amplitude of its oscillations immediately after inflation can be as large as $0.1 M_{P}$.

In this case we again have a situation when mode function of heavy fermions has WKB form between zeros of $M_{\text {eff }}$, and can be described with parabolic scattering around this instance. Therfore our general formula (32) works for superheavy fermions as well. The only modification is that the phase between successive scatterings will be defined by the integral over time intervals between them. In formula (32) parameter $\Lambda_{k}(\tau)$ will be

$$
\Lambda_{k}(\tau)=-\frac{\pi \kappa^{2}}{a^{2} \sqrt{\frac{q_{0}}{a^{3}}-\tilde{m}^{2}}}
$$


In case of massive fermions, the criteria for excitation instead of (35) will be

$$
\frac{\kappa_{s}^{2}}{a^{2} \sqrt{\frac{q_{0}}{a^{3}}-\tilde{m}^{2}}} \leq 1 / \pi
$$

At the start of background oscillations for large $q_{0}$ a term $\tilde{m}^{2}$ is negligible. Thus, as in the last section, we expect the Fermi sphere to fill with the radius $\kappa_{s}=\sqrt{q_{0}} a^{1 / 4}$. However, as $q$ drops, equation (37) solved for $\kappa_{s}^{2}$ reaches a maximum, $\kappa_{m}$. and then decreases. This maximum occurs when the scale factor is $a_{m}=\left(\frac{q_{0}}{\tilde{\tilde{m}}^{2}}\right)^{1 / 3} \cdot$ This gives $\kappa_{m}^{2}=\frac{\sqrt{3}}{\pi}\left(\frac{q_{0}}{4 \sqrt{\tilde{m}}}\right)^{2 / 3}$. Notice that $k_{m}$ scales as $q_{0}^{1 / 3}$. This is still not the final width of the band. This is because there are a number of background oscillations which occurs between the moment $a_{m}$ and the moment when denominator in (36) approaches zero. We put a number of oscillation before $a_{m}$ and between $a_{m}$ and the moment of zero denominator as approximately equal and equal to $N_{m}$. In this time after $a_{m}$ even the modes suppressed exponentially as $e^{-\Lambda_{k}}$ can achieve significant occupation numbers in their random stochastic walk. We found the amplification factor in the from of the exponent is $\sqrt{N_{m}}$. In contrary to the ligh fermions case, there is no perturbative end of the process. Excitation of superheavy fermions is abruptly terminated when $\frac{q_{0}}{a^{3}}-\tilde{m}^{2}$.

We found the spectrum of created superheavy particles after the process is terminated is given by formula

$$
n_{k}=\frac{1}{2} \exp \left(-2 \frac{\left(\kappa-\gamma \kappa_{m}\right)^{2}}{\kappa_{m}^{2}}\right)
$$

where $2 \gamma^{2}=\ln \left(\frac{q_{0}}{4 \pi^{2} \tilde{m}^{2}}\right)$. This formula is valid for $N_{m}$ greater than a few. In Fig. 11 we plot numerically calculated final spectrum of the supermassive fermions vs. an analytic formula 38, which are in a good agreement. In the case of superheavy particles in an expanding universe the maximum radius of the k-sphere is scaled with $q_{0}$ as $\gamma k_{m} \sim q_{0}^{1 / 3} \ln q_{0}$, which is in close agreement with [8]. However, one shall also check that at the moment when excitation of superheavy fermions terminates, their back reaction $h\langle\bar{\psi} \psi\rangle$ to the dynamics of inflaton oscillation is still negligible.

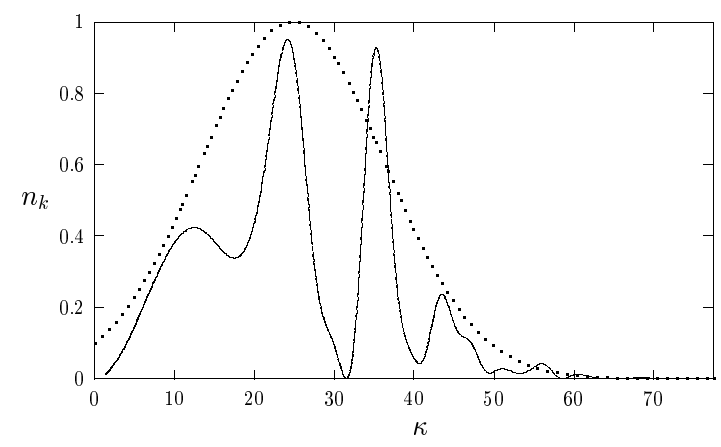

FIG. 11. Final spectrum of heavy fermions with masses $m_{\psi}=50 m_{\phi}$ when $q_{0}=10^{6}$. Also shown is the analytic estimate (38).

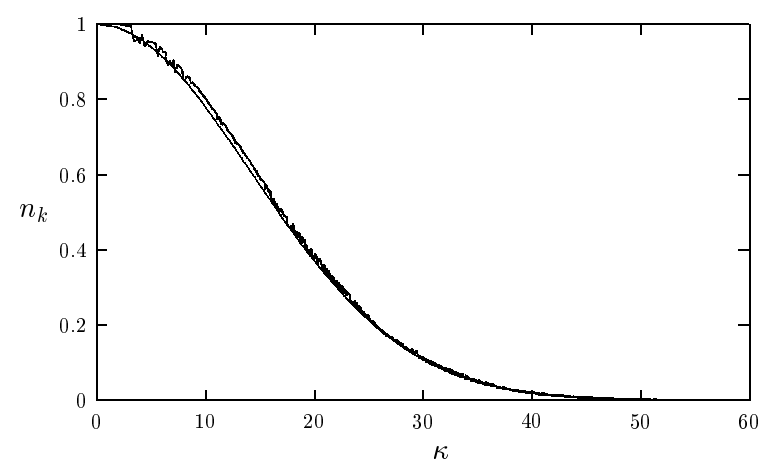

FIG. 12. The comoving occupation number $n_{k}$ of fermions after $M_{\text {eff }}$ crosses one zero for $q=10^{6}$ and $\tilde{m}=50$. Also shown is the analytic formula (35) which fits perfectly.

In Fig. 11 we plot spectrum of superheavy fermions after the process is terminated. Dashed curves are obtained with analytic formula (38).

\section{Fermionic Production from Single Kick}

As we seen formula (32) can be extended to the case of expanding universe. Consider the very first term in formula (32). As It corresponds to the creation of fermions after effective mass $M_{e f f}(t)$ crosses zero for the first time. In this case we found 


$$
n_{k}=e^{-\pi \Lambda_{k}^{2}}
$$

Occupation number of fermions generated from the single kick is plotted in Fig. 12. Two curves corresponding to the formula (39) and to the numerical solutions are shown and practically indistinguishable.

\section{DISCUSSION}

In this paper we developed a non-perturbative theory for fermion production by an oscillating inflaton field. As we have seen, the production of fermions can be characterized as parametric excitation. Even in the simple model of a Yukawa coupling between fermions and a background scalar field in an expanding universe oscillating around the minimum of its quadratic potential, the theory of fermionic parametric excitation is rich and leads to important results. Fermions are created very quickly, within about ten(s) oscillations, in out-of-equilibrium states. For large values of the resonance parameter $q=\frac{h \phi_{0}}{m_{\phi}}$, occupation number of light fermions $\left(m_{\psi}<m_{\phi} \ll h \phi_{0}\right)$ is changing in a step-like manner at instances $t_{j}$ when the inflaton amplitude $\phi\left(t_{j}\right)=0$ passes through zero, $j=1,2,3, .$. We have developed the method of parabolic scatterings for fermions, based closely on a similar approach for the bosonic resonance [2]. It is possible to derive a unified recursive formula, which relates the occupation number of fermions or bosons $n_{k}^{j+1}$ at the moment $t_{j+1}$ to the earlier value $n_{k}^{j}$ :

$$
\begin{aligned}
n_{k}^{j+1} & =e^{-\pi \Lambda_{k}^{2}}+\left(1 \pm 2 e^{-\pi \Lambda_{k}^{2}}\right) n_{k}^{j} \\
& -2(-1)^{j} e^{-\frac{\pi}{2} \Lambda_{k}^{2}} \sqrt{1 \pm e^{-\pi \Lambda_{k}^{2}}} \sqrt{n_{k}^{j}\left(1 \pm n_{k}^{j}\right)} \sin \theta_{t o t}^{j},
\end{aligned}
$$

For bosons one shall use an upper sign and neglect the $(-1)^{j}$ terms [2], while for fermions one shall use the lower sign. For light fermions and bosons $\Lambda_{k}^{2}=\frac{k^{2}}{\sqrt{q} m_{\phi}^{2}}$. For large $q$ the angle $\theta_{t o t}^{j}$ can be treated as a random phase. As a result, formula (40) predicts the stochastic character of parametric excitation of both bosons and fermions. In the fermionic case it leads to the conclusion that in the momentum space fermions chaotically fill up a broad sphere of the radius $\sim q^{1 / 4} m$. This formula also clearly shows the features of: spontaneous emission: for both bosons and fermions, the first oscillation leads to the spectrum $n_{k}=e^{-\pi \Lambda_{k}}$; stimulated emission for bosons with $n_{k} \gg 1$, we have $\left(n_{k}^{j+1}-n_{k}^{j}\right) \propto n_{k}^{j}$; Pauli Blocking for fermions, if $n_{k}^{j}=1$, the next value will always be $n_{k}^{j+1}=1-e^{-\pi \Lambda_{k}}$ even in the stochastic case. This prevents the occupation number of fermions from exceeding 1.

Formula (40) can be extended to the case of massive bosons and fermions. However, here important differences emerge. For bosons we will have $\Lambda_{k}=\frac{k^{2}+m_{b}^{2}}{\sqrt{q} m_{\phi}}$, where $m_{b}$ is the $\chi$-boson mass. It leads to the conclusion that the creation of superheavy, $m_{b}>m_{\phi}$, bosons is exponentially suppressed. However, for fermions we have $\Lambda_{k}=$ $\frac{k^{2}}{m_{\phi}^{2} s q r t h^{2} \phi_{0}^{2}-m_{\psi}^{2}}$. Therefore even superheavy fermions with the mass as large as $h \phi_{0}$ can be created in abundance from the coherent inflaton oscillations [7, 8]. This occurs because the effective mass of fermions is given by the algebraic combination $h \phi(t)+m_{\psi}$ and the creation of fermions occurs when the effective mass goes to zero. The method of parabolic scatterings, applied for instances when this happens, leads to both formulae (40) with the corresponding $\Lambda_{k}$.

There are situations where single instance (single kick) of particle creation may lead to interesting effects. An example is the scenario of instant preheating, which is especially important for inflationary models without minima of the inflaton potential [15]. Another example is the interaction of the inflaton with superheavy fermions during the inflationary stage when the combination $h \phi(t)+m_{\psi}$ can go through zero only once. The generic formula (40) embraces the case where bosons and fermions are created by a single kick. We shall put $j=0, n_{k}^{0}=0$, and then $n_{k}^{1}=e^{-\pi \Lambda_{k}^{2}}$. However, instead of the parameter $q$ we shall use another combination of coupling constants and $\phi_{0}$ and $m_{\phi}$. In this case the effect is defined by the velocity $\dot{\phi}_{*}$ at the moment $t_{*}$, which is different for bosons and fermions. For bosons single instance creation of particles gives the spectrum [2.15]

$$
n_{k}=e^{-\frac{\pi\left(k^{2}+m_{\chi^{2}}\right)}{g \phi_{*}}}
$$

where $t_{*}$ corresponds to $\phi\left(t_{*}\right)=0$. For fermions single instance creation gives [14]

$$
n_{k}=e^{-\frac{\pi k^{2}}{h \phi_{*}}}
$$

where $\phi\left(t_{*}\right)+m_{\psi} / h=0$. 
We believe that the theory of fermionic preheating will be important ingredient of the realistic scenarios of the reheating of the universe after inflation.

Acknowledments. Collaboration and discussions with Andrei Linde and Alexei Starobinsky significantly influenced this work. We also thank Jügen Baacke for useful discussion. This work was supported by NSERC and CIAR.

[1] L. A. Kofman, A. D. Linde, and A. A. Starobinsky, Phys. Rev. Lett. 73, 3195 (1994); Y. Shtanov, J. Trashen, R. Brandenberger, Phys. Rev. D51, 5438 (1995); D. Boyanovsky, H. J. deVega, R. Holman, D.S. Lee, A. Singh, Phys. Rev. D51, 4419 (1995).

[2] L. A. Kofman, A. D. Linde, and A. A. Starobinsky, Phys. Rev. D56, 3258 (1997).

[3] S. Khlebnikov \& I. Tkachev, Phys. Rev. Lett. 77219 (1996).

[4] J. Baacka, K. Heitmann \& C. Pätzold, Phys.Rev. D58, 125013 (1998); hep-ph/9806205

[5] P. Greene, L. Kofman, Phys. Lett. B448, 6 (1999), hep-ph/9807339.

[6] D. Boyanovsky, M. D'Attanasio, H. J. de Vega, R. Holman, \& D.S. Lee, Phys. Rev. D52, 6805 (1995).

[7] P. Greene, in: Cosmo98, November 1998; AIP Proceedings, v. 478, Ed. D. Caldwell, pp.72-74; hep-ph/9905256

[8] G. Giudice, M. Peloso, A. Riotto, I. Tkachev; JHEP 9908, 014 (1999); 9905242

[9] P. B. Greene, L. Kofman, A. D. Linde, and A. A. Starobinsky, Phys. Rev. D56, 6175 (1997)

[10] M.V. Mostepanenko and V.M. Frolov, Sov. J. Nucl. Phys., 19, 885 (1974); A. A. Grib, S. G. Mamayev, and V. M. Mostepanenko, Vacuum Quantum Effects in Strong Fields (Friedmann Laboratory, St. Petersburg, 1994).

[11] R. Kallosh, L. Kofman, A. Linde and A. Van Proeyen, hep-th/9907124; Phys. Rev. D, in press; G.F. Giudice, I. Tkachev, A. Riotto, hep-ph/990751d, JHEP 9908:009,1999 .

[12] J. Garcia-Bellido, S. Mollerach, E. Roulet, hep-ph/0002076

[13] J. Baacke, C. Patzold, hep-ph/9912505

[14] D. Chung, E. Kolb, A. Riotto, I. Tkachev, hep-ph/9910437.

[15] G. Felder, L. Kofman, A. Linde, Phys. Rev.D59, 1999, 123523; hep-ph/9812289 